\title{
INTERVENÇÃO PSICOLÓGICA PARA PACIENTES ATENDIDOS NO PROGRAMA DE OBESIDADE DO AMBULATÓRIO DOCENTE-ASSISTENCIAL DA BAHIANA (PEPE'): UM RELATO DE EXPERIÊNCIA
} \author{
Amanda S. F. de Cerqueira $\begin{aligned} & \text { Psicóloga graduada pela Escola Bahiana de } \\ & \text { Medicina e Saúde Pública (2010). Pós- }\end{aligned}$ \\ graduanda em Psicoterapia Cognitivo- \\ Comportamental pela WP - Psicoterapia \\ Cognitivo-Comportamental.
}

Maria de Lourdes Lima de Souza e Silva

Médica Endocrinologista, Doutora em Medicina e Saúde pela Universidade Federal da Bahia (2008). Professora Adjunta da graduação, pós-graduação e Coordenadora do Ambulatório de Obesidade da Escola Bahiana de Medicina e Saúde Pública.

\section{Sylvia Maria Barreto da Silva}

Psicóloga graduada pela Universidade Federal da Bahia (1994). Mestranda em Medicina e Saúde Humana (Escola Bahiana de Medicina e Saúde Pública2013). Docente dos Cursos de Psicologia, Enfermagem e Responsável Técnica pelo Serviço de Psicologia da Escola Bahiana de Medicina e Saúde Pública.

\begin{abstract}
Resumo
O texto corresponde a um relato de experiência sobre o processo de intervenção psicológica para pacientes atendidos no Programa de Obesidade do Ambulatório Docente-assistencial da Bahiana. O presente estudo fornece informações sobre tratamento psicológico em grupo na abordagem da Terapia CognitivoComportamental (TCC) com pacientes que possuem o diagnóstico clínico de obesidade. O objetivo geral do programa de intervenção psicológica foi possibilitar uma melhoria na qualidade de vida dos pacientes através de intervenções multidisciplinares. Como objetivos específicos pretendeu-se fornecer informações aos pacientes por meio da psicoeducação sobre aspectos etiológicos, cognitivos, emocionais e socioculturais que envolvem a obesidade; trabalhar estratégias de enfrentamento para a adesão à dietoterapia e outras abordagens terapêuticas; identificar e promover a modificação de pensamentos e comportamentos disfuncionais acerca dos hábitos alimentares e estilo de vida; investigar a presença de sintomas ansiosos e/ou depressivos durante o percurso do tratamento. Aponta que a intervenção psicológica, com base na Terapia Cognitivo-comportamental, no tratamento da obesidade, contribui positivamente na ampliação da adesão do tratamento da obesidade.
\end{abstract}

Palavras-chave: Intervenção psicológica; Obesidade; Terapia Cognitivo-comportamental.

\footnotetext{
${ }^{\text {i }}$ Projeto de Estudo do Excesso de Peso. 


\section{INTRODUÇÃO}

A obesidade é um dos problemas mais relevantes de saúde pública na atualidade, uma vez que é um fator de risco que pode desencadear diversas doenças. ${ }^{(1)}$ Trata-se de uma doença crônica crescente mundialmente e, em decorrência da sua abrangência, vem configurando-se quase como uma epidemia. (2) Sendo um fenômeno complexo e multifacetado, a sua compreensão demanda análise e integração de diversos saberes científicos oriundos de diferentes profissões, como a Psicologia e a Psiquiatria. ${ }^{(3,4)}$ Todavia, no DSM-R não há critérios para inclusão da obesidade na categoria de transtornos alimentares, embora os obesos também apresentem perturbações comportamentais e psíquicas relacionadas à alimentação. ${ }^{(5)}$

O sinal preponderante da obesidade é o excesso de gordura corporal, sendo que diversos estudos epidemiológicos apontam para a correlação entre essa doença, diversas comorbidades orgânicas, níveis de mortalidade e morbilidade somática, apontando para a necessidade de estudos aprofundados das possíveis causas e consequências desse problema. ${ }^{(1-3)}$

O diagnóstico da obesidade é obtido basicamente de duas formas. Diagnóstico quantitativo, no qual é utilizado o cálculo do Índice de Massa Corporal (IMC) e, assim, é considerado obeso o indivíduo que apresenta o IMC igual ou superior a $30 \mathrm{Kg} / \mathrm{m}^{2}$. O diagnóstico qualitativo que se refere à distribuição de gordura no corpo, classificado como andróide quando o acúmulo é na parte superior ou abdominal, ou ginecóide quando o é na parte inferior ou subcutâneo. $^{(2,3)}$

Por um lado, o diagnóstico fornece informações importantes que traduzem a urgência pelo tratamento e norteiam a escolha por uma intervenção terapêutica. Por outro, para definir a melhor conduta terapêutica é necessário conhecer os fatores causais da obesidade. É consenso entre os estudiosos que a origem e o desenvolvimento da obesidade é multifatorial. ${ }^{(3)}$

A obesidade advém, tanto de desequilíbrios bioquímicos e de doenças físicas, como de sintomas e perturbações de ordem psicológica e, consequentemente, dificuldade de adaptação. ${ }^{(1)}$ Em nível psicológico a alteração da imagem corporal pelo ganho de peso pode provocar desvalorização da autoestima, do autoconceito e, como consequência, pode acarretar sintomas ansiosos e/ou depressivos, diminuição da sensação de bem-estar, sentimento de inadequação social, enfim, contribuir de forma negativa para a qualidade de vida do sujeito. ${ }^{(1)}$ Problemas emocionais geralmente são percebidos como consequência da obesidade, mas 
podem preceder a doença. A ansiedade e a depressão são quadros comuns, sendo que a depressão maior costuma acometer em maior escala a obesidade mórbida. ${ }^{(4)}$

No nível físico, biológico, utilizam-se comumente abordagens terapêuticas objetivando mudança de comportamento e de estilo de vida, propondo estratégias como programas de dietas, atividades físicas, e em alguns casos, medicamentos. ${ }^{(3)}$ Entretanto, como a obesidade é complexa, torna-se difícil modificar o estilo de vida, as práticas alimentares e todo um sistema de crenças intrínsecas que perpassam o modo de operar do sujeito, somente por meio de abordagens terapêuticas que incidem sobre o aspecto biológico do paciente obeso. Segundo Vasques, ${ }^{(4)}$ o uso de medicação nem sempre é a primeira alternativa. Antes deve compor o tratamento multidisciplinar. A dietoterapia associada à psicoterapia, por ser menos invasiva, deve ser sempre priorizada.

Por sua complexidade, faz-se necessário uma compreensão da obesidade que contemple os aspectos psicológicos já que influenciam sobremaneira a doença em que o sintoma manifesto é o engordar. ${ }^{(3)}$ Dessa forma, é imprescindível a intervenção psicológica associada a outras abordagens de uma equipe multidisciplinar, a fim de potencializar a eficácia dos objetivos de um programa voltado ao tratamento da obesidade. A atuação psicológica nesse tratamento é de suma importância, pois esse profissional dispõe de recursos terapêuticos fundamentais e diferenciados como a escuta ativa, a relação empática, técnicas de manejo cognitivo e comportamentais, bem como instrumentos psicométricos de uso exclusivo do psicólogo. Todos esses recursos atrelados à leitura do sujeito para além do biológico, isto é, para os conteúdos latentes presentes no curso da obesidade, fazem da intervenção psicológica um componente necessário e favorável junto a uma equipe multidisciplinar.

Nesse contexto, a intervenção psicológica é necessária, uma vez que, quanto maior o IMC mais problemas psicológicos são apresentados pelos pacientes obesos. ${ }^{(6)}$ Estes, além do peso da gordura, sofrem no nível emocional, devido às dificuldades, limitações e sofrimento. ${ }^{(7)} \mathrm{O}$ psicoterapeuta junto ao tratamento da obesidade, mais do que seu papel de cientista, tem o papel de artista, pois, em última instância, atua auxiliando na mudança da visão de mundo, na opinião do obeso e, isso é uma forma de criar. O psicólogo "lança mão" do recurso da informação, a fim de que o paciente aceite sua condição de obeso e possa optar pelo recurso terapêutico indicado pelo médico no seu caso. ${ }^{(8)}$ 


\section{CONTEXTUALIZAÇÃO DA EXPERIÊNCIA}

O presente estudo fornece informações sobre tratamento psicológico em grupo na abordagem da Terapia Cognitivo-Comportamental (TCC) com pacientes que possuem o diagnóstico clínico de obesidade. O objetivo geral do programa de intervenção psicológica foi possibilitar uma melhoria na qualidade de vida dos pacientes através de intervenções multidisciplinares. Como objetivos específicos pretendeu-se fornecer informações aos pacientes por meio da psicoeducação sobre aspectos etiológicos, cognitivos, emocionais e socioculturais que envolvem a obesidade; trabalhar estratégias de enfrentamento para a adesão à dietoterapia e outras abordagens terapêuticas; identificar e promover a modificação de pensamentos e comportamentos disfuncionais acerca dos hábitos alimentares e estilo de vida; investigar a presença de sintomas ansiosos e/ou depressivos durante o percurso do tratamento.

Essa experiência relatada ocorreu no Serviço de Psicologia da Escola Bahiana de Medicina e Saúde Pública (SEPSI). Os pacientes foram recrutados por meio do encaminhamento realizado a partir da triagem médica do Serviço de Endocrinologia do Ambulatório Docente-Assistencial da Bahiana (ADAB).

Como critério de inclusão para ambos os grupos, os pacientes deveriam ter o diagnóstico de obesidade sem distinção de grau da doença; apresentar dificuldade de adesão à dietoterapia; adultos; ambos os sexos; ter disponibilidade para manter-se no tratamento psicoterapêutico até o término. Já no caso de pacientes portadores de comorbidades de humor ou ansiedade entendidas como mais relevantes no momento do que a obesidade; pacientes com diagnóstico de fobia social para falar em público; analfabetos; com demanda para tratamento psicológico individual; desinteressados em aderir à terapia na abordagem de grupo, foram excluídos do programa.

O universo trabalhado foi formado por dois grupos A e B. O primeiro, composto por quatro pacientes, realizado entre os meses de outubro, novembro e dezembro de 2010. O segundo, por sete pacientes, entre os meses de setembro, outubro e novembro e dezembro de 2011. Estes números retratam os pacientes que completaram as sessões, pois em ambos os grupos, duas pacientes abandonaram por motivos pessoais. No primeiro grupo, propusemos uma formação com até oito pacientes e, no segundo, até doze pacientes, tendo como base protocolos de atendimento em grupo que nos serviram de inspiração metodológica.

A metodologia utilizada foi inspirada em protocolos de atendimento em grupo na abordagem da terapia cognitivo-comportamental, sendo os mesmos adaptados e redesenhados 
em consonância com os objetivos do programa. O grupo A foi o primeiro grupo realizado e o B foi o segundo.

Esse programa foi estruturado em três etapas. Etapa de pré-tratamento que consistiu em uma triagem por meio da qual foi possível "rastrear" a problemática vivenciada por cada paciente; esclarecer brevemente a modalidade terapêutica a partir desse momento e estabelecer um contrato de continuidade ou não no grupo.

A triagem foi realizada em dois momentos. Primeiro foi feita uma entrevista inicial para avaliação geral do problema (Anamnese de história de vida), na qual foram investigados dados da história de vida do paciente, seu desenvolvimento, o aparecimento da obesidade e sua evolução, a forma e as estratégias de se relacionar com o alimento, a presença de sintomas de transtornos alimentares como comer compulsivo e bulimia. Na sessão seguinte de triagem, foi realizada a entrevista estruturada para Transtornos do Eixo $\mathrm{I},{ }^{(9)}$ para investigação mais aprofundada de dados relativos a transtornos como depressão maior, transtorno de pânico, fobias, transtorno obsessivo- compulsivo, transtorno de estresse pós-traumático, transtorno de ansiedade generalizada, bulimia e anorexia, dentre outros.

A segunda etapa foi nomeada como tratamento. O protocolo desenvolvido para o grupo A consistiu em oito sessões, realizadas uma vez por semana com duração de uma hora e trinta minutos. O protocolo foi modificado e o grupo B consistiu em quinze sessões, realizadas uma vez por semana com duração de uma hora e trinta minutos a duas horas. Nesta fase, no grupo A, foram utilizados três instrumentos para avaliação da amostra nesses grupos. Inventário Beck de Depressão (BDI). ${ }^{(10)}$ Inventário Beck de Ansiedade (BAI) ${ }^{(11)}$ e Questionário de Qualidade de Vida SF-36. ${ }^{(12)}$ Estes instrumentos foram selecionados, pois trazem dados importantes concernentes com os objetivos do programa, bem como tomamos conhecimento de que um vasto número de pesquisas e artigos científicos publicados faz uso desses instrumentos que são validados no país. No grupo $B$, adicionamos mais um instrumento, sendo este qualitativo, a dieta definitiva de Beck - Questionário. ${ }^{(13-14)}$

A terceira etapa, pós-tratamento, conhecido como follow-up, consta de sessões de acompanhamento. Para ambos os grupos, propusemos a realização de três sessões com intervalos de quatro meses durante o período de um ano. Estas sessões são importantes, pois contribuem para verificar e reforçar a manutenção de ganhos e a prevenção de recaídas.

Nesse momento, as sessões de tratamento serão apresentadas em duas tabelas, como componente metodológico relevante, com vistas a contribuir com o fomento de novas 
Cerqueira et al. Intervenção psicológica para pacientes atendidos no programa de obesidade do ambulatório docente-assistencial da Bahiana (PEPE ): um relato de experiência

propostas de tratamento bem sucedidas a serem disponibilizadas para essa população que carece de estudos e atenção da Psicologia.

\section{Roteiro Sumarizado do Protocolo de Tratamento da Obesidade em Oito Sessões (Grupo A)}

Primeira Sessão

- Recepção dos pacientes

- Preenchimento das escalas (BDI, BAI, SF-36)

- Informações sintéticas sobre o que é a obesidade, etiologia, curso da doença, tratamento

- Informações sobre a Terapia Cognitivo-Comportamental e o modelo cognitivo

- Firmação do contrato terapêutico

- Tarefa de casa

Segunda Sessão

- Revisão da tarefa de casa

- Incentivo ao automonitoramento (diário alimentar)

- Incentivo ao controle de estímulos

- Aprendendo a gerenciar a alimentação fora de casa, em férias e ocasiões especiais

- Tarefa de casa

Terceira Sessão

- Revisão da tarefa de casa

- Discussão das preocupações com o tratamento (prós e contras de comprometerse)

- Utilização do checklist de obstáculos para a perda de peso

- Técnica de resolução de problemas em seis passos

- Tarefa de casa

Quarta Sessão

- Educação nutricional (palestra da nutricionista)

- Tarefa de casa 
Cerqueira et al. Intervenção psicológica para pacientes atendidos no programa de obesidade do ambulatório docente-assistencial da Bahiana (PEPE ): um relato de experiência

Quinta sessão

- Revisão da tarefa de casa

- Diferenciando o conceito de imagem corporal e aparência física

- Avaliação para identificação de preocupações com a imagem corporal (lista de preocupações com a imagem corporal)

- Psicoeducação sobre o desenvolvimento, manutenção e maneiras de modificar

- Uma imagem corporal negativa

- Autopercepção da imagem corporal (figuras de perguntas de identificação ou distanciamento)

Sexta Sessão

- Revisão da tarefa de casa

- Monitoração das preocupações com a imagem corporal (R.P.D.)

- Aumento das atividades físicas em geral

- Benefícios do aumento das atividades físicas

- Identificação da meta de peso para emagrecer (distinção de peso ideal, desejado e possível)

- Questionando o peso desejado

- Benefícios da perda de peso moderada (10\% a $15 \%)$

- Tarefa de casa

Sétima sessão

- Revisão da tarefa de casa

- Continuando a trabalhar a imagem corporal, identificando e corrigindo os vieses de percepções e interpretações

- Enfatizando a necessidade de aceitação e mudança

- Tarefa de casa

Oitava sessão

- Revisão da tarefa de casa

- Reaplicação das escalas iniciais (BDI, BAI, SF-36)

- Aprendendo sobre os objetivos independentes da perda de peso

- Identificando objetivos primários

- Administrando os objetivos primários 
- Revisando o aprendizado de manejo cognitivo e comportamental aprendido e conquistas com a terapia.

- Avaliação do tratamento

- Informações sobre a sessão de acompanhamento (prevenção de recaídas)

\section{Roteiro Sumarizado do Protocolo de Tratamento da Obesidade em Quinze Sessões (Grupo B)}

Primeira Sessão

- Recepção dos pacientes

- Preenchimento das escalas (BDI, BAI, SF-36, Questionário Beck)

- Informações sintéticas sobre o que é a obesidade, etiologia, curso da doença, tratamento.

- Informações sobre a Terapia Cognitivo-Comportamental e o modelo cognitivo

- Firmação do contrato terapêutico

- Tarefa de casa

Segunda Sessão

- Revisão da tarefa de casa

- Influências do pensamento sobre a dificuldade de adesão à dieta

- Aprendendo que o comportamento de comer não é automático

- Aprendendo sobre os gatilhos que precedem a alimentação

- Diferenças de pensamento: pessoas magras X pessoas obesas

- Tarefa de casa

Terceira Sessão

- Revisão da tarefa de casa

- Aprendendo a identificar pensamentos sabotadores

- Trabalhando os pensamentos sabotadores: técnica da paciência

- Questionamento da crença da injustiça

- Trabalhando a motivação: vantagens de emagrecer

- Cartões de enfrentamento para combate da desmotivação

- Tarefa de casa 
Quarta Sessão

- Revisão da tarefa de casa

- Importância do autoelogio

- Conhecendo erros cognitivos comuns à pessoa obesa

- Questionamento de erros cognitivos com a técnica das sete perguntas

- Aprendendo a organizar ambientes para facilitar a adesão ao tratamento

- Tarefa de casa

Quinta sessão

- Educação nutricional (palestra da nutricionista para pacientes e familiares)

- Orientações sobre a importância do apoio social

- Tarefa de casa

Sexta Sessão

- Revisão da tarefa de casa

- Incentivo ao controle de estímulos

- Importância de sentar para comer e comer devagar e conscientemente

- Importância das atividades físicas e da manutenção

- Aprendendo a planejar horários para dieta e atividade física

- Incentivo ao automonitoramento (diário alimentar)

- Tarefa de casa

Sétima Sessão

- Revisão da tarefa de casa

- Diferenciação: fome, vontade e desejo incontrolável de comer

- Modificando a definição de saciedade

- Aprendendo a tolerar a fome

- Superando o desejo incontrolável por comida - técnica de distração

- Tarefa de casa

Oitava Sessão

- Revisão da tarefa de casa

- Utilização do checklist de obstáculos para a perda de peso

- Evitação da alimentação não planejada

- Aprendendo a acabar com excessos alimentares 
- Aprendendo a resolver problemas

- Tarefa de casa

Nona Sessão

- Revisão da tarefa de casa

- Técnica de resolução de problemas em seis passos

- Aprendendo a gerenciar a alimentação fora de casa, em férias e ocasiões especiais

- Aprendendo a resistir ao gatilho social

- Tarefa de casa

Décima Sessão

- Revisão da tarefa de casa

- Consumo de bebida alcoólica

- Aprendendo a eliminar a alimentação emocional

- Diferenciando o conceito de imagem corporal e aparência física

- Avaliação para identificação de preocupações com a imagem corporal (lista de preocupações com a imagem corporal)

- Tarefa de casa

Décima Primeira Sessão

- Revisão da tarefa de casa

- Psicoeducação sobre o desenvolvimento, manutenção e maneiras de modificar

- Uma imagem corporal negativa

- Autopercepção da imagem corporal (figuras de perguntas de identificação ou distanciamento)

- Monitoração das preocupações com a imagem corporal (R.P.D.)

- Tarefa de casa

Décima Segunda Sessão

- Revisão da tarefa de casa

- Continuando a trabalhar a imagem corporal, identificando e corrigindo os vieses de percepções e interpretações

- Identificação da meta de peso para emagrecer (distinção de peso real, ideal e possível) 
- Questionando o peso desejado

- Benefícios de uma perda de peso moderada (10 a 15\%)

- Enfatizando a necessidade de aceitação e mudança

- Tarefa de casa

Décima Terceira Sessão

- Revisão da tarefa de casa

- Aprendendo sobre os objetivos independentes da perda de peso

- Identificando objetivos primários

- Administrando objetivos primários

- Trabalhando a autoconfiança

- Tarefa de casa

Décima Quarta Sessão

- Revisão da tarefa de casa

- Diminuição do estresse em três passos

- Manutenção da atividade física

- Estímulo à construção de nova lista de tarefas

- Descobrindo o momento de parar de emagrecer e começar a manter

- Manutenção do novo peso

- Tarefa de casa

Décima Quinta Sessão

- Reaplicação das escalas iniciais (BDI, BAI, SF-36, Questionário Beck)

- Revisão da tarefa de casa

- Revisando o aprendizado de manejo cognitivo e comportamental aprendido e conquistas com a terapia.

- Avaliação do tratamento

- Informações sobre a sessão de acompanhamento (prevenção de recaídas)

- Fechamento da etapa de tratamento "lanche saudável".

\section{RESULTADOS E DISCUSSÃO}

A construção do primeiro protocolo composto por oito sessões foi embasada na Terapia Cognitivo-Comportamental para os pacientes atendidos no ADAB. Foi um projeto 
esquematizado com poucos achados na literatura sobre a contribuição da Psicologia em termos de estudos e técnicas a serem trabalhadas com esse público. Diante dessa realidade, baseamos a elaboração do protocolo à luz de referências ${ }^{(15-17)}$ e fundamentos de cunho mais comportamental do que cognitivo.

A amostra do grupo A era composta por um homem e três mulheres, sendo três componentes com obesidade e, uma, com sobrepeso. O homem não apresentava comorbidades, já entre as mulheres, uma havia sofrido de episódio depressivo, uma sofria de depressão maior e outra de depressão leve atual e traços de fobia social de falar em público. Ao iniciarmos o tratamento, os pacientes apresentavam-se ansiosos e motivados, entretanto desconfiados sobre a possibilidade de a abordagem psicológica auxiliá-los na melhora dos comportamentos relacionados à alimentação frente a um histórico longo que cada um percorreu de tentativas fracassadas em outros tratamentos para perda de peso e manutenção de ganhos.

No decorrer do tratamento, os pacientes foram se engajando diante dos conteúdos e técnicas trabalhadas, realizando, na maioria das sessões, a tarefa de casa. Foi notório que uma das pacientes do sexo feminino e um do masculino se beneficiavam mais, pois eram os que mais participavam trazendo contribuições, dúvidas e na assiduidade em cumprir as tarefas de casa. Sem dúvida, o ganho mais significativo revelado pelo grupo foi a partir do momento em que foram estimulados a realizar semanalmente, a cada dia, como tarefa de casa, o automonitoramento alimentar, por meio do registro no diário alimentar.

Outro fator marcante na educação dos pacientes e mudança comportamental foi sinalizado com a contribuição da palestra da nutricionista. Esta palestra foi realizada para pacientes e familiares. No tocante à técnica mais cognitiva relacionada ao contexto da imagem corporal, foi interessante perceber que ao contrário da literatura, neste grupo apenas uma paciente sofria devido a não aceitação do corpo, bem como distorção de imagem corporal. Outro dado relevante refere-se ao fato de esse grupo ter tido uma integrante com sobrepeso. Esta não revelou benefícios tão notórios quanto os outros membros e, diante do trabalho com algumas temáticas e mesmo técnicas, não se identificava ao certo com aquele contexto.

Ao término do grupo, os pacientes revelaram por meio dos registros e falas que se surpreenderam com os aprendizados, sobretudo por terem mantido o autocontrole diante da alimentação, por terem despertado a percepção para os objetivos primários, para além da fonte de prazer oral, melhorado a autoconfiança e autoestima. Solicitaram também como espaço de sugestão que fosse acrescentado para grupos futuros mais sessões de tratamento. Entretanto, 
os pacientes não emagreceram com esta proposta do protocolo, mas também não engordaram, mantiveram apenas o peso do início do tratamento.

Em relação à fase pós-tratamento, devido a vários fatores de estruturação cronológica, as sessões de acompanhamento não foram realizadas com esse grupo. O retorno breve foi realizado por meio do contato telefônico com dois pacientes e uma paciente foi encaminhada para realizar cirurgia de redução do estômago. Estes contatos foram realizados em menos de dois meses após o término do grupo e verificou-se que os ganhos foram mantidos.

O protocolo para o grupo B foi modificado após avaliação realizada por nossa equipe do primeiro grupo. Nesse sentido, diante dos achados, percebemos a necessidade primordial de complementar o protocolo inicial com embasamento mais cognitivo, já que no primeiro grupo foi mais comportamental. As pesquisas foram redirecionadas e uma janela do trabalho foi solucionada a partir da inserção de técnicas cognitivas, como relatadas por Beck. ${ }^{(13-14)}$

A amostra deste grupo era de sete pacientes, todas do sexo feminino. As pacientes apresentavam certa homogeneidade de comportamentos e critérios diagnósticos que eram similares. Já haviam apresentado pelo menos sintomas importantes de depressão e ansiedade acentuada, na maioria, neste caso, não configurada como transtorno. Apresentavam longo histórico de tentativas fracassadas no tratamento clínico da obesidade. Todas demonstravam certa dúvida quanto à eficiência e eficácia da Psicologia no tratamento clínico.

Nesse protocolo, ampliado e modificado para quinze sessões, a estruturação realizada seguiu uma organização lógica. Até a quarta sessão, aproximadamente, o foco do tratamento foi sobre a identificação, desafio de pensamentos sabotadores e erros cognitivos comuns às pessoas que sofrem com excesso de peso. Foram utilizadas, nesse momento, técnicas mais focadas na cognição (pensamentos), o que possibilitou sobremaneira a flexibilidade cognitiva e posterior mudança de comportamento.

A partir desse ponto, as pacientes começaram a verbalizar que estavam entendendo o porquê de tantas tentativas sem sucesso, pois ainda não tinham tido a oportunidade de trabalhar com o que consideraram a raiz dos problemas: pensamentos sabotadores. A cada sessão participavam com depoimentos interessantes, sobre gatilhos que precediam a alimentação, como a postura dos seus familiares, a identificação e atenção para não ceder aos pensamentos sabotadores, o aprendizado com o respeito aos limites por meio da técnica da paciente, o que reflete um engajamento do grupo e do foco no trabalho cognitivo nesse início.

A palestra da nutricionista marca um tratamento com o foco mais cognitivocomportamental. O recurso do automonitoramento com registro do diário alimentar mais uma 
vez mostrou-se eficaz na medida em que nos escritos das pacientes tornou-se clara a diminuição do consumo de alimentos não planejados e/ou não saudáveis. Todas foram cada uma em seu momento apropriando-se e aderindo mais corretamente a proposta alimentar orientada pela nutricionista ainda antes do ingresso no grupo. Outro fator de grande relevância diz respeito à adesão de todas as pacientes à atividade física. Todas realizaram caminhada conforme rotina acordada no grupo terapêutico, com exceção de uma delas devido a comorbidades da obesidade nos membros inferiores.

Houve destaques com ganhos individuais. Duas pacientes, por exemplo, que mais ingeriam bebida alcoólica, diminuíram o consumo. Outra, após a primeira sessão do grupo, matriculou-se na academia, prática de que não gostava antes, mas passou a fazê-lo com motivação e assiduidade. Uma paciente com diagnóstico de fobia social para falar em público, ao longo do tratamento foi se encorajando, assumindo uma postura cada vez mais ativa e verbalizando melhoras explícitas no tocante à autoestima e melhorias em outras áreas da vida.

Uma paciente, no início do grupo, sofreu um luto pelo falecimento da filha. Antes de completar uma semana deste evento, realizamos a sessão semanal e logo a paciente relatou o caso. Neste dia a sessão foi modificada e ela foi acolhida pelo grupo e, depois, individualmente pela terapeuta. Neste momento revelou que, mesmo diante do sofrimento, achou importante não faltar à sessão, já que estava se beneficiando. Outra paciente vivenciou um luto com uma tia e escolheu vir para a sessão da terapia ao invés de viajar para a cidade onde foi realizado o sepultamento. Informou que o processo grupal foi fundamental nesta escolha, pois relevou que, em outro momento, teria paralisado sua rotina, mas dessa vez não estava disposta a fazer isto, pois acreditava que precisava priorizar a sua saúde naquele momento.

A partir da sétima sessão, foi interessante o fato de uma paciente emagrecer $10 \mathrm{~kg}$, sendo que já havia iniciado o emagrecimento antes do ingresso no nosso grupo. Até o término das sessões, foi surpreendente a fala das pacientes. Algumas tinham tido consulta de retorno com a nutricionista ou com a endocrinologista do programa e outras encorajaram-se e subiram na balança em diferentes locais. A constatação foi que, ao final desta fase de tratamento, todas as pacientes relataram que emagreceram. Com o ingresso no nosso grupo até o término, com quinze sessões, conforme relatos, o mínimo de emagrecimento foi cerca de $4 \mathrm{~kg}$ e o máximo de perda conquistada, a partir do grupo, foi de $8,600 \mathrm{~kg}$.

Além disso, diante da sessão final de avaliação, as pacientes revelaram quão importante foi para elas realizar um tratamento psicológico e consideraram, de forma unânime, que outras pessoas precisam ter acesso a esse tipo de tratamento, pois reconhecem 
que seu conteúdo e condução foram o despertar, o gatilho para a mudança que precisavam nesse percurso difícil do controle de peso que, até então, não conseguiram somente com tratamento médico e nutricional.

A fase de acompanhamento deverá acontecer durante um ano, após o término do grupo, com intervalo de quatro meses.

\section{CONSIDERAÇÕES FINAIS}

O presente estudo possibilitou fazer um breve levantamento na literatura acerca do contexto das pessoas que sofrem da condição de obesidade. Deparamo-nos com a dificuldade de encontrar um número importante de artigos sobre tratamento psicológico para esse público na abordagem da Terapia Cognitivo-Comportamental. Diante dessa realidade, a maior parte de ambos os protocolos foi baseada em apenas dois livros.

No tocante aos grupos A e B realizados, o discurso dos pacientes e a mudança de comportamento observadas no curso do tratamento revelam que o primeiro grupo careceu de um enfoque mais cognitivo, de um engajamento maior dos participantes em aderir mais concretamente às propostas como, por exemplo, a atividade física.

Já no grupo B, com a ampliação das sessões por meio da inclusão de um trabalho inicial voltado para a cognição, somada à ampliação de mais técnicas comportamentais, formou-se um grupo mais motivado e engajado e obtido um resultado expressivo e inovador na vida desses participantes.

De acordo com os achados supracitados diante da análise qualitativa dos grupos, percebemos que a Terapia Cognitivo-Comportamental foi eficiente e eficaz nesses tratamentos, principalmente, no segundo grupo em que esta combinação terapêutica foi trabalhada claramente.

Este estudo apresenta limitações, por se tratar de uma aplicação em uma dada unidade de tratamento e, portanto, não termos o comparativo em outros locais para possível generalização sobre a eficácia desse protocolo. Também, o tratamento dos dados quantitativos advindos dos instrumentos aplicados na fase de tratamento não foi concluído e, sendo assim, este estudo baseou-se na análise qualitativa e não quali-quanti. Enfim, esperamos contribuir com este estudo para o fomento de novos tratamentos ou mesmo sua ampliação, objetivando uma melhor assistência junto à equipe multidisciplinar que trabalha com pacientes com obesidade. 


\section{REFERÊNCIAS}

1. Silva MP da, Jorge Z, Domingues E, Lacera Nobre E, Chambel P, Castro J de. Obesidade e qualidade de vida. Acta Med Port [periódicos na Internet]. 2006 [acesso em 3 set 2010]; 19:247-50. Disponível em: http://www.actamedicaportuguesa.com/pdf/2006-19/3/247250.pdf.

2. Mancini MC. Noções fundamentais diagnóstico e classificação da obesidade. In: Garrido Junior AB. Cirurgia da obesidade. São Paulo: Atheneu; 2004; p. 1-7.

3. Benedetti C. De obeso a magro: a trajetória psicológica. São Paulo: Vetor; 2003. Capítulo 1. Obesidade: diagnóstico, etiologia e tratamento; p. 21-49.

4. Vasques F, Martins FC, Azevedo AP de. Aspectos psiquiátricos do tratamento da obesidade. Rev psiquiatr clín. [periódicos na Internet]. 2004 [acesso em 6 set. 2010]; 31: 195-8. Disponível em: http://www.scielo.br/pdf/rpc/v31n4/22408.pdf.

5. Flaherty D, Janicak PG. Psiquiatria, diagnóstico e tratamento. Porto Alegre: Artes Médicas; 1995.

6. Cordás TA. Participação do psiquiatra e do psicólogo na fase perioperatória. Aparticipação do psiquiatra. In: Garrido Junior AB. Cirurgia da obesidade. São Paulo: Atheneu; 2004. p. 71-4.

7. Franques ARM. Participação do psiquiatra e do psicólogo na fase perioperatória. Bparticipação do psicólogo. In: Garrido Junior AB. Cirurgia da obesidade. São Paulo: Atheneu; 2004. p. 75-9.

8. Marchesini SD. Distúrbios psíquicos e obesidade In: Garrido Junior AB. Cirurgia da obesidade. São Paulo: Atheneu; 2004. p. 25-33.

9. Amorim P. Mini International Neuropsychiatric Interview (MINI): validação de entrevista breve para diagnóstico de transtornos mentais. Rev Bras Psiquiatr. 2000; 22:10615 .

10. Beck AT, Ward CH, Mendelson M, Mock J, Erbaugh J. An inventory for measuring depression. Arch Gen Psychiatry 1961; 4:561-71.

11. Beck AT, Epstein N, Brown G, Steer RA. An inventory for measuring clinical anxiety: psychometric properties. J Consult Clin Psychol. 1988; 56(6): 893-7.

12. Ciconelli RM, Ferraz MB, Santos W, Meinão I, Quaresma MR. Tradução para a língua portuguesa e validação do questionário genérico de avaliação de qualidade de vida SF-36 (Brasil SF-36). Rev Bras Reumatol. 1999; 39:143-50.

13. Beck JS. Pense magro: a dieta definitiva de Beck. Porto Alegre: Artmed; 2009.

14. Beck JS. Livro de tarefas pense magro: programa de seis semanas da dieta definitiva de Beck. Porto Alegre: Artmed; 2009. 
Cerqueira et al. Intervenção psicológica para pacientes atendidos no programa de obesidade do ambulatório docente-assistencial da Bahiana (PEPE ): um relato de experiência

15. Sociedade Brasileira de Endocrinologia e Metabologia. Obesidade: terapia cognitivocomportamental. [Internet]. 2005 [acesso em 6 set. 2010]; p. 1-10. Disponível em: http://www.projetodiretrizes.org.br/5_volume/34-ObesTerap.pdf

16. Cooper Z, Fairburn CG, Hawker, DM. Terapia cognitivo-comportamental da obesidade: manual do terapeuta. São Paulo: Roca; 2009.

17. Fernandes MLM, Vieira C, Gomes VFM, Nogueira D, Silva FA da. Ambulatório interdisciplinar para o tratamento da obesidade em pacientes com compulsão alimentar: projeto [Internet]. [2004] [acesso em 6 set. 2010]; p. 1-9. Disponível em:

http://www.Scribd.Com/doc/21745622/ambulatorio-interdisciplinar-para-o-tratamento-daobesidade-em- pacientes-com-compulsao-alimentar. 